\title{
Biocharacterization of Heat Shock Protein 90 in Acetaminophen-Treated Livers Without Conspicuous Drug Induced Liver Injury
}

\author{
$\mathrm{Ka} \mathrm{Wu}^{\mathrm{a}}$ Chao Guo ${ }^{\mathrm{b}}$ Min Su${ }^{\mathrm{c}}$ Xinmou Wu${ }^{\mathrm{d}}$ Rong Lic \\ aDepartment of Pharmacy, The Second People's Hospital of Nanning City, The Third Affiliated Hospital \\ of Guangxi Medical University Nanning, Guangxi, 'D Department of Pharmacy, Guigang City People's \\ Hospital, The Eighth Affiliated Hospital of Guangxi Medical University, Guigang, 'Key Laboratory of \\ Tumor Immunology and Microenvironmental Regulation, Guilin Medical University, Guangxi, Guilin, \\ ${ }^{\mathrm{d} C o l l e g e ~ o f ~ P h a r m a c y, ~ G u a n g x i ~ M e d i c a l ~ U n i v e r s i t y, ~ G u a n g x i, ~ P R ~ C h i n a ~}$
}

\section{Key Words}

Acetaminophen • Hsp90 • Biomarker • Liver injury

\begin{abstract}
Background/Aims: Acetaminophen (APAP) refers to a medication used to manage pain and fever symptoms. Heat shock protein 90 (Hsp90) is to be expressed during various stresses, such as wound healing and tissue remodeling. Recently, it is discovered that Hsp90 is a potential modifier of cytogenesis. In comparison to clinical references of liver damage, this study was designed to assess the potential bioeffect of Hsp90 in APAP-treated livers without conspicuous drug induced liver injury (DILI). Methods: In our current study, human plasma samples of APAP-used patients were collected for biochemical assays in clinical parameters. Adult male mice were used to investigate the biocharacterization of Hsp90 in APAP-treated livers through serological tests and immunoassays. Further, a mouse liver cell strain was employed in assessment of bioeffect of APAP on hepatocellular Hsp90 expression. Results: Correspondingly, the clinical data showed APAP-administered patients resulted in increased Hsp90 levels in serum when compared to other clinical parameters of liver injury. In adult mice study, APAP-treated livers showed unchanged hepatocellular and metabolic functions, as highlighted in biochemical analysis and immunoassay. Notably, Hsp90 expression in APAPtreated mice were elevated in the serum and liver samples. In quantitative western blot assay, the present data suggested that hepatocellular Hsp90 level was up-regulated followed by APAP treatment. In mouse cell strain study, APAP-treated liver cells had increased trend of aminotransferase contents and apoptotic counts. Further, endogenous Hsp90 expression in APAP-exposed cells was increased dose- and time-dependently. Conclusions: In conclusion, our current findings disclose that Hsp90 biomolecule may be a potential indicator for APAPinduced inconspicuous DILI, in which it seems to be characterized with more sensitive than other diagnosis criteria.

K. Wu and C. Guo contributed equally to this work.




\section{Introduction}

A liver injury implies sustained lesion to liver cells, gradually resulting in hepatic dysfunctions [1]. Chemical-induced liver damage, especially drug-induced liver injury (DILI), is a leading cause of acute and chronic liver disease in clinical practice [2]. Certain common medicinal agents, when taken in overdoses and even within therapeutic ranges, may impair the liver cells [3]. Some medications often cause potential damage to the liver, manifesting only as abnormal liver enzyme level. And DILI is responsible for $5 \%$ of all hospital patients and $50 \%$ of all acute liver failure cases, such as acetaminophen (APAP) [4]. Commonly, APAP is believed to be a safe drug in use for antipyretic and analgesic treatments, and it has the side effects time- or dose-dependently, including gastrointestinal reaction and hepatic damage [5]. APAP-induced hepatotoxicity accounts for the majority of acute liver impairment in community cases when APAP treatment. Generally, superthreshold use of APAP in adult can be identified as $4000 \mathrm{mg}$ /body per day [6]. In biological toxicology analysis, APAP-medicated hepatotoxicity can be originated from its toxic metabolites in body, which these intermediate products can in turn destroy the liver function [7]. Instead, officially recommended APAP dosage is deemed to be harmless, and thus APAP is a common self-service medication in daily life [8]. However, the assessment of APAP-induced early liver impairment before clinical diagnosis remains uninvestigated. Biologically, production of high level of heat shock protein can be triggered by exposure to different kinds of environmental stress conditions, such as infection, inflammation, toxin induction [9]. Hsp90 is involved in activation of steroid receptor and transcription factor, showing as a promising anti-apoptotic effector [10]. This consequence means that Hsp90 may play potential bioeffect on early liver impairment. In this study, we evaluated the potential application of determining Hsp90 as an attractive biomarker for detecting inconspicuous liver damage induced by APAP exposure through detectable data from the clinical, animal and cellular experiments.

\section{Materials and Methods}

\section{Drug and materials}

Acetaminophen (APAP) used in clinical study was purchased from Sinopharm Group Guangdong MediWorld Pharmaceutical Co., Ltd. (Foshan, China). And APAP (HPLC $\geq 99 \%$ ) used in vivo and vitro studies was purchased from Shanghai YuanYe Biological Technology Co., Ltd (Shanghai, China). In addition, other corresponding materials/reagents were marked in respective experimental sections.

\section{Clinical design}

A total of 30 patients (male: female $=1: 1$ ), aged 25 to 33 years old, were recruited when they experienced a common cold prior to consuming APAP. All participants were physically examined and clinically diagnosed to exclude the persons who were using medication, hyperpyrexia (high fever), or had infectious diseases, metabolic dysfunctions, and liver diseases. In consequence, $10 \mathrm{APAP}$-used patients were identified in this study. Meanwhile, other 10 participants (aged 21 to 30 years) were set as APAP-free controls after being validated without liver damage through clinically diagnosed. Blood samples were aseptically harvested from the elbow vein at 2 - and 26 -hour time points. The plasma was immediately stored at $-20^{\circ} \mathrm{C}$ for further tests by using an automatic blood analyzer (Hitachi, Japan). In this study, all subjects signed the informed consent forms prior to human blood collection. In addition, the clinical procedures were conducted according to the Ethical Guidelines of the Declaration of Helsinki [11].

\section{Cell culture}

Mouse liver cell strain (AML12) were purchased from Chinese Academy of Sciences Typical Culture Collection (Shanghai, China). When arrival, cells were cultured with 10\% fetal bovine serum (GIBCO, USA)$1 \%$ penicillin/streptomycin (Solarbio, Beijing, China)- DMEM-high glucose medium (Solarbio, Beijing, China), and incubated in a $5 \% \mathrm{CO}_{2}$ incubator at $37^{\circ} \mathrm{C}$. Following by treatment with APAP $(2.5 \mathrm{mM}$ and $5 \mathrm{mM})$ for $2 \mathrm{~h}$ and $26 \mathrm{~h}$, the supernatants and cells were harvested to conducting biochemical tests and immunoassays. 


\section{Cellular Physiology Cell Physiol Biochem 2017;43:1562-1570 \begin{tabular}{ll|l} 
DOI: 10.1159/000482003 & $\begin{array}{l}\text { O 2017 The Author(s). Published by S. Karger AG, Basel } \\
\text { www.karger.com/cpb }\end{array}$
\end{tabular}}

Wu et al.: Bioeffect of Hsp90 on APAP-Treated Liver

\section{Animal study}

Adult male Kunming mice, aged 6-7 weeks old, were provided by the Laboratory Animal Centre of Guilin Medical University. This animal study was approved by the Institutional Ethical Committee of Guilin Medical University (No. GLMC201603002). All mice were housed in plastic cage, under the controlled conditions of temperature $\left(24 \pm 2^{\circ} \mathrm{C}\right)$ and cycled light $(12 \mathrm{~h}$ light $/ 12 \mathrm{~h}$ dark).

The mice were acclimatized for at least 1 week when arrival. And then APAP-exposed mice were intragastrically administered with single and twice doses of APAP solution in saline buffer ( $300 \mathrm{mg} / \mathrm{kg}$ ), and other untreated mice were treated with the same volume of saline solution. All mice were killed via cervical dislocation at $2 \mathrm{~h}$ and $26 \mathrm{~h}$ APAP exposures. And sera were stored immediately at $-80^{\circ} \mathrm{C}$ when collection. In addition, tissues of interest were harvested for further biochemical tests.

\section{Chemical assay}

At the end of experiments, human plasma and mice serum/liver specimens were collected as previously described [12]. All samples were subjected to deproteinization before high performance liquid chromatography (HPLC) determination, which commercially available APAP standard (Yuanye Biology Co. Ltd. Shanghai, China) was set as a reference control. HPLC apparatus (LC-20A, Shimadzu, Japan) equipped with a SIL-20AC detector and ASTON RG C18 column (4.6 mm $\times 50 \mathrm{~mm}$ i.d., $5 \mu \mathrm{m}$; China) was conducted to measure the APAP contents. A working mobile phase with the ratio of acetonitrile: isopropanol: purified water is 23:8:69 in triethylamine solution $(\mathrm{pH}=6.9)$ was at designed flow speed of $1.1 \mathrm{ml} / \mathrm{min}$. As results, detectable APAP contents were normalized and expressed as final $\mu \mathrm{g} / \mathrm{ml}$ or $\mu \mathrm{g} / \mathrm{mg}$ protein.

\section{Biological test and cytohistological staining}

A series of functional biomolecules was determined in human plasma and mice/cell samples through using automatic analyzer and spectrophotometer, respectively. In addition, human Hsp90 and mouse Hsp90, insulin, glucagon, triglyceride and free fatty acid were calculated with commercially available kits (Shanghai Elisa Biotech Co., Ltd., China) by using enzyme linked immunosorbent assay (ELISA) method.

Mouse liver, pancreas, kidney tissues were fixed with 4\% paraformaldehyde (PFA) before being cut as $5 \mu \mathrm{m}$ paraffin sections. And then the section was subjected to routine hematoxylin and eosin staining (Boster, Wuhan, China). Meanwhile, liver section was further conducted to immunofluorescence staining. In brief, the section was blocked with $5 \%$ BSA buffer for $1 \mathrm{~h}$, and then incubated with primary antibody of Hsp90 (Beyotime Biotechnology, China) for overnight at $4^{\circ} \mathrm{C}$, followed by incubation with IgG H\&L (Alexa Fluor $₫ 488$ ) (Abcam, UK) for $1 \mathrm{~h}$ at room temperature. Subsequently, DAPI dye (Abcam) was used to nuclear staining prior to imaged and data analysis [13].

\section{Immunohistochemical staining}

Mouse liver, pancreas, kidney sections were dewaxed and rehydrated, and then blocked with 5\%BSA in PBS-Tx-100 $/ 0.05 \%$ for $1 \mathrm{~h}$ at room temperature. Subsequently, the sections were incubated with hepatocyte growth factor (HGF) (1:150, Bioss, China), insulin (1:500, Santa Cruz, USA), renin (1:150, Bioss, China) primary antibodies overnight at $4^{\circ} \mathrm{C}$. After being washed with PBSTx buffer several times, the sections were exposed to corresponding antibodies (1:500, Boster, China), and then re-exposed to hydrogen peroxidase enzyme-labeled diaminobenzidine (DAB) dye before re-dyeing with haematoxylin to cytoblast $[14,15]$.

\section{Western blot test}

Mouse liver protein was extracted by using RIPA buffer plus 1mM PMSF protein inhibitor (Beyotime Biotechnology, China). And $40 \mu \mathrm{g}$ protein/lane (liver tissue) and $10 \mu \mathrm{g}$ protein/lane (cell strain) were separated with $10 \%$ SDS-PAGE, and then was blotted to $0.2 \mu \mathrm{m}$ PVDF membrane (Millipore, MA, USA). After being blocked with 5\% non-fat milk buffer (Yili Industrial Group Co., Ltd, China) for $1 \mathrm{~h}$ at room temperature, membrane was incubated with diluted Hsp90 (1:500, Beyotime, China), AKR7A (1:1000, Santa Cruz, USA), Bcl-2 (1:500, Beyotime, China), p44/42MAPK (1:1000, Beyotime, China), Actin (1:500, Beyotime, China) primary antibodies overnight at $4{ }^{\circ} \mathrm{C}$, followed by HRP-coupled secondary antibody (1:2000, Beyotime, China) for $1 \mathrm{~h}$ at room temperature. After incubating with chemiluminescent agent, the membrane was developed and imaged under Imager System (Bio-Rad, USA) [16]. 


\section{Cellular Physiology Cell Physiol Biochem 2017;43:1562-1570 \begin{tabular}{ll|l} 
and Biochemistry Published online: October 16, 2017 & $\begin{array}{l}\text { C } 2017 \text { The Author(s). Published by S. Karger AG, Basel } \\
\text { www.karger.com/cpb }\end{array}$ \\
\hline
\end{tabular}

Table 1. The diagnostic parameters of APAP-used patients, Ctrl, control; APAP, acetaminophen; TBIL, total bilirubin; DBIL, direct bilirubin; IBIL, indirect bilirubin; ALT, glutamic-pyruvic transaminase; AST, glutamic-oxaloacetic transaminase; ALP, alkaline phosphatase; GGT, glutathione transpeptidase; TBA total, bile acids; 5-NT, 5-nucleotide enzyme; TP, total protein; ALB, albumin; GLP, globulin; Hsp90, heat shock protein 90; UD, undetected. Note: ${ }^{\mathrm{a}} \mathrm{P}<0.05$ vs Ctrl (=control)

Statistical analysis

Statistical data were assayed by using statistical product and service solutions (SPSS) 19.0 software. Differences between two comparable groups were assessed by a one-way analysis of variance (ANOVA) followed by Student's t test. Processed data were expressed as mean \pm SD, in which $\mathrm{p}<0.05$ was considered as statistically significant.

\begin{tabular}{lccc}
\hline Markers & Ctrl & APAP(2h) & APAP(26h) \\
\hline TBIL(U/L) & $9.5 \pm 1.3$ & $9.2 \pm 2.1$ & $8.8 \pm 1.6$ \\
DBIL $(\mu \mathrm{mol} / \mathrm{L})$ & $3.3 \pm 1.4$ & $2.9 \pm 0.5$ & $2.9 \pm 0.9$ \\
IBIL $(\mu \mathrm{mol} / \mathrm{L})$ & $6.0 \pm 1.1$ & $6.2 \pm 1.6$ & $5.9 \pm 0.8$ \\
ALT(U/L) & $16.2 \pm 2.4$ & $17.2 \pm 8.3$ & $18.8 \pm 7.1$ \\
AST(U/L) & $17.0 \pm 3.7$ & $21.6 \pm 4.6$ & $21.2 \pm 4.4$ \\
ALP (U/L) & $77.2 \pm 6.9$ & $83.6 \pm 7.9$ & $86.6 \pm 10.0$ \\
GGT (U/L) & $19.3 \pm 2.7$ & $21.4 \pm 4.6$ & $22.4 \pm 5.1$ \\
TBA ( $\mu \mathrm{mol} / \mathrm{L})$ & $5.7 \pm 0.9$ & $6.2 \pm 1.9$ & $6.7 \pm 1.9$ \\
5-NT (U/L) & $2.5 \pm 1.0$ & $3.3 \pm 0.8$ & $3.5 \pm 0.8$ \\
TP (g/L) & $66.4 \pm 8.1$ & $76.1 \pm 3.5$ & $74.7 \pm 2.8$ \\
ALB (g/L) & $47.1 \pm 4.3$ & $51.8 \pm 1.8$ & $51.5 \pm 1.5$ \\
GLP (g/L) & $19.3 \pm 4.1$ & $24.3 \pm 3.7$ & $23.2 \pm 3.3$ \\
Blood-APAP( $\mu \mathrm{g} / \mathrm{ml})$ & $\mathrm{UD}$ & $15.46 \pm 3.51$ & $16.71 \pm 2.89$ \\
Blood-Hsp90(pg/mL) & $68.51 \pm 3.24$ & $98.76 \pm 5.14$ & $154.49 \pm 8.25^{\mathrm{a}}$ \\
\hline
\end{tabular}

\section{Results}

Clinical hallmark of diagnostical biomolecules in APAP-used patients

As revealed in HPLC data, the visible APAP contents in plasma of APAP-used patients showed as $(10.23 \pm 0.42)$ and $(9.29 \pm 0.70) \mu \mathrm{g} / \mathrm{ml}$ at 2 and 26 hours, respectively. These data suggested current detectable level of APAP was under effective chemotherapeutical concentration in the body. Further data from Table 1 exhibited that APAP-treated patients resulted in non-significant statistic difference on liver functional enzymes when compared to APAP-free controls or clinical references $(P>0.05)$. However, plasma Hsp90 content in APAP-used patients was greater than that in untreated controls $(P<0.05)$ at two respective time-points.

\section{Investigation of metabolic functions in APAP-exposed mice}

In order to observe APAP-induced metabolic functions, designed dosage of APAP was exposed to mice for further investigation. As results, there were no comparable data of body/liver weights and liver index (P > 0.05) between APAP-exposed and APAP-free mice (Fig. 1A). As highlighted in routine HE staining, unchanged cellular morphology and cytoarchitecture in liver, pancreas, and kidney tissues were observed in these grouped mice. In addition, immunohistochemical assay showed biomarkers of target tissue were normally expressed, characterized with plenty of HGF-, insulin-, renin-positive cells. (Fig. 1B). Further, immunoblotting results suggested that cell death-indicated biomolecules of AKR7A, Bcl-2, and $\mathrm{p} 44 / 42 \mathrm{MAPK}$ in the liver showed insignificance between the grouped mice $(P>0.05$; Fig. 1C). As detailed in Table 2, representative metabolic parameters (blood glucose, insulin, lipids, pancreatic amylase, BUN, LDH), liver functional enzymes (blood ALT, AST, AKP, TS) between compared groups showed no significant differences $(P>0.05)$. Compared to untreated mice, serum and liver Hsp90 contents in APAP-treated mice was elevated, particularly in $26 \mathrm{~h}$ dosed mice $(P<0.05)$. 
Fig. 1. Bioeffect of APAP on mouse liver functions. (A) The detectable data showed there were unchanged significantly of body and liver weights between APAP-dosed and untreated mice. (B) As showed in HE staining, unchanged cell morphology and architecture in the liver, pancreas, and kidney tissues were observed in these grouped mice. In addition, immunohistochemical assay showed biomarkers of target tissue were normally expressed, characterized with plenty of HGF-, insulin-, renin-
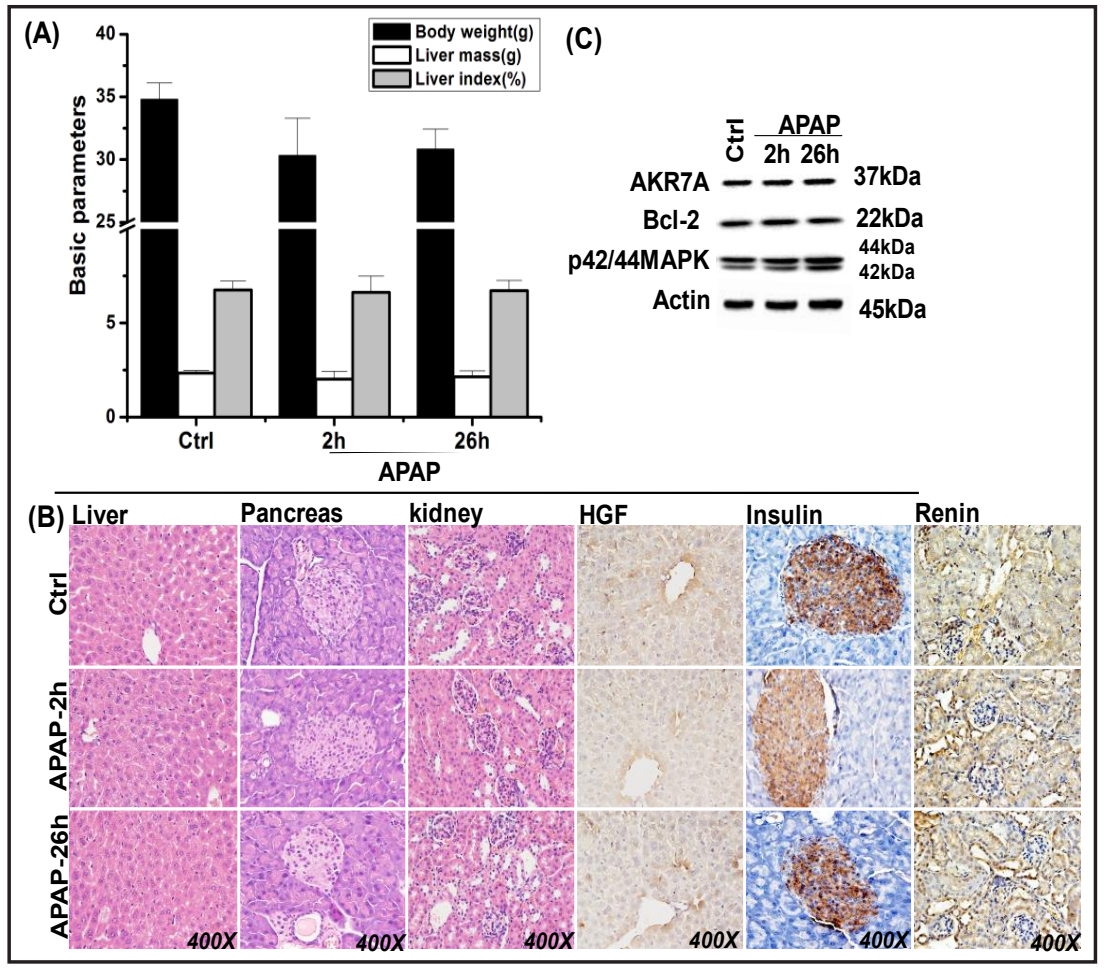
positive cells (Magnification 400×). (C) Results from western blot indicated that cell death-indicated biomolecules of AKR7A, $\mathrm{Bcl}-2$, and $\mathrm{p} 44 / 42 \mathrm{MAPK}$ in the liver resulted in insignificance between the grouped mice.

Table 2. The biological parameter in APAP-dosed mice, Ctrl, control; APAP, acetaminophen; HDL-C, high-density lipoprotein cholesterol; LDL-C, lowdensity lipoprotein cholesterol; T-CHO, total cholesterol; TG, triglyceride; ALT, glutamic-pyruvic transaminase; AST, glutamic-oxaloacetic transaminase; AKP, alkaline phosphatase; LDH, lactate dehydrogenase; TS, trypsinase; BUN, urea nitrogen; Hsp90, heat shock protein 90; UD, undetected. Note: ${ }^{\text {a }}<0.05$ vs Ctrl (=control)

Bioeffect of APAP exposure on hepatic Hsp90 in mice

Further, we aimed to characterize hepatic immunophenotype of Hsp90 in APAP-treated mice, specific immunostaining was performed. As revealed in histocytological analysis (immunohistochemistry and immunofluorescence), APAPexposed liver had more Hsp90-positive cells than that in untreated liver, especially in $26 \mathrm{~h}$ dosed treatment $(P<0.05)$ (Fig. 2A). For further validation, quantitative data from western blot showed that APAP-treated liver resulted in up-regulated expression of Hsp90 dose-dependently, in which $26 \mathrm{~h}$ dosed liver had greater level of Hsp90 than that in APAP-free liver $(P<0.05)$ (Fig. 2B).

\begin{tabular}{lccc}
\hline Markers & Ctrl $(26 \mathrm{~h})$ & APAP $(2 \mathrm{~h})$ & APAP $(26 \mathrm{~h})$ \\
\hline Blood glucose(mmol/mL) & $5.72 \pm 0.86$ & $7.38 \pm 1.18$ & $7.60 \pm 0.47$ \\
Insulin (mIU/L) & $21.07 \pm 0.34$ & $21.94 \pm 0.59$ & $22.14 \pm 0.16$ \\
HDL-C (mmol/g prot) & $0.92 \pm 0.06$ & $0.86 \pm 0.01$ & $0.95 \pm 0.12$ \\
LDL-C (mmol/g prot) & $1.71 \pm 0.19$ & $1.73 \pm 0.06$ & $1.80 \pm 0.20$ \\
T-CHO (mmol/g prot) & $0.13 \pm 0.01$ & $0.15 \pm 0.03$ & $0.16 \pm 0.01$ \\
TG (mmol/g prot) & $1.59 \pm 0.11$ & $1.43 \pm 0.04$ & $1.61 \pm 0.08$ \\
ALT(U/L) & $31.79 \pm 1.77$ & $44.76 \pm 5.98$ & $56.02 \pm 5.92$ \\
AST(U/L) & $57.62 \pm 8.10$ & $67.73 \pm 6.73$ & $79.58 \pm 7.47$ \\
AKP(U/L) & $38.57 \pm 2.83$ & $42.92 \pm 4.88$ & $50.02 \pm 1.89$ \\
BUN(mmol/L) & $6.69 \pm 0.89$ & $6.66 \pm 0.90$ & $7.86 \pm 0.87$ \\
TS(U/ml) & $793.60 \pm 92.54$ & $874.20 \pm 94.88$ & $894.87 \pm 80.57$ \\
LDH(U/L) & $430.18 \pm 46.17$ & $432.15 \pm 33.05$ & $407.61 \pm 90.35$ \\
Blood-APAP( $\mu$ g/ml) & $\mathrm{UD}$ & $112.37 \pm 12.22$ & $96.71 \pm 6.53$ \\
Blood-Hsp90(pg/mL) & $52.87 \pm 1.81$ & $77.14 \pm 3.88$ & $104.24 \pm 1.37 \mathrm{a}$ \\
Liver- Hsp90 (pg/mg) & $86.09 \pm 4.16$ & $129.39 \pm 3.39$ & $188.28 \pm 9.39 \mathrm{a}$ \\
& & & \\
\hline
\end{tabular}


Fig. 2. Characterization of Hsp90 expression induced by APAP in mouse liver. APAP-dosed livers exhibited elevated Hsp90-positive cell counts, as highlighted in immunohistochemistry and immunofluorescencent stains (Magnification 400×). Meanwhile, western blot results suggested that APAP-dosed liver resulted in up-regulated Hsp90 expression time-dependently. Statistical data were assayed by using one-way ANOVA followed by Student's t test, and the data was expressed as the mean \pm SD. Note: vs. Ctrl (=control), ${ }^{\mathrm{a}} \mathrm{P}<0.05$.
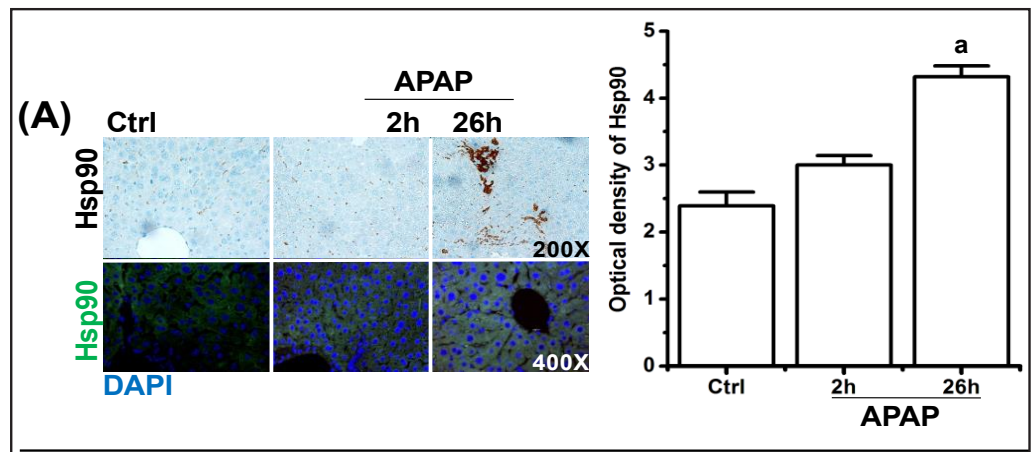

(B)

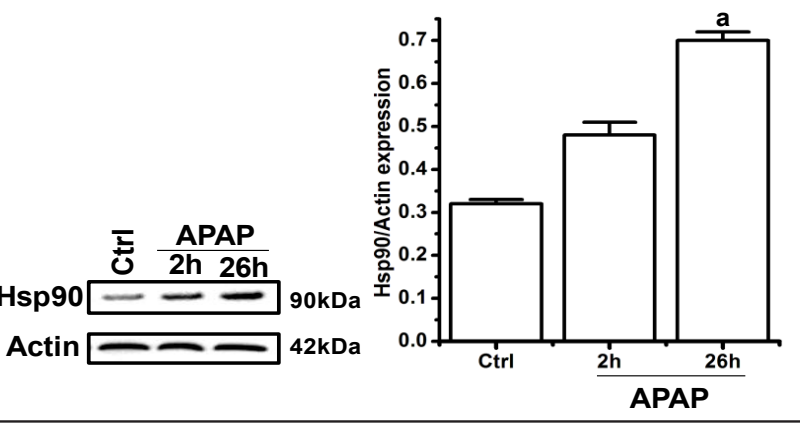

Fig. 3. Bioeffect APAP on Hsp90 expression in mouse liver cells. (A) APAP-exposed mouse liver cells resulted in increased trend of transaminase enzymes (ALT, AST). (B) In validation in immunohistochemistry obesrvations (Magnification 400×), increased Hep90-

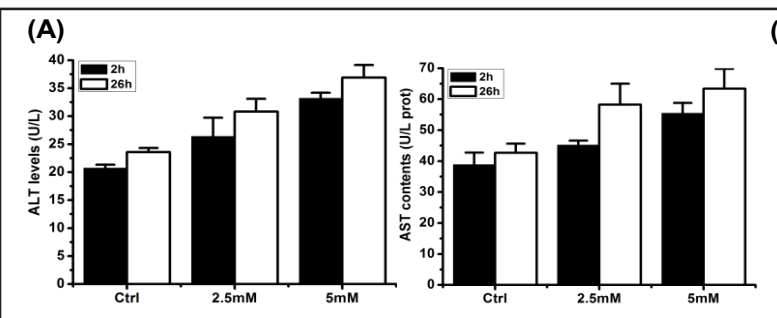

(C)
(B) Ctrl

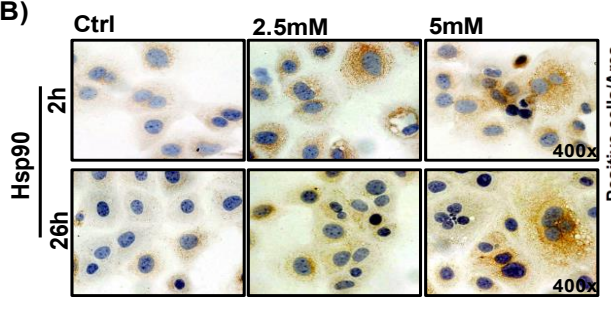

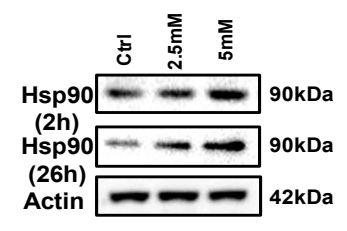

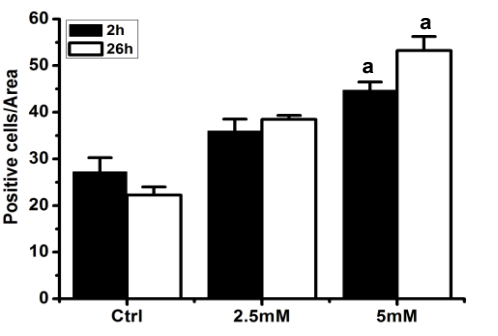

positive cells were

observed in APAP-dosed cells, especially in $26 \mathrm{~h}$ dosed cells. (C) Data from western blot showed that hepatic Hep90 content in APAP-treated cells was upregulated dose-dependently when compared to that in control. Statistical data were assayed by using one-way ANOVA followed by Student's t test, and the data was expressed as the mean \pm SD. Note: vs. Ctrl $\left(=\right.$ control), ${ }^{\text {a }}<0.05$.

\section{Bioeffect of APAP exposure on hepatic Hsp90 in vitro}

We next investigate the immediate effect of APAP on Hsp90 expression by using a mouse liver cell (AML12). In consequence, mouse liver cells-produced transaminase contents (ALT, AST) were showed increased trend of expression in the supernatants after being exposed to 2.5, 5mM APAP for respective 2, 26 hours (Fig. 3A). In addition, increased Hep90-positive cells were observed in APAP-exposed liver cells, especially in $26 \mathrm{~h}$ dosed cells $(P<0.05)$ (Fig. 3B). Validated data from immunoblotting exhibited that hepatocellular Hep90 level in APAPtreated cells was upregulated dose-dependently when compared to that in control (Fig. 3C). 


\section{Discussion}

Acetaminophen (APAP), commonly used in antipyretic or analgesic remission, is found to be involvement of side effects in overdose, characterized with time- or dosedependent liver impairment in officially recommended dosage. In toxicological mechanism, APAP-induced hepatocellular impairment can be originated from its metabolite toxin of $\mathrm{N}$-acetyl-p-benzoquinonimine which impairs to liver cells in turn [17-19]. In recent years, ever-increasing evidences have showed APAP induced visible liver injury as elucidated in proposed biological signaling pathways and molecular mechanisms [20, 21]. However, APAP-induced early liver dysfunction is still less investigated in effective identification. Therefore, development of potential biomarker of screening early liver lesion by APAP may be a promising strategy to reduce APAP-associated health risk. Our current data indicated that APAP-dosed patients resulted in inconspicuous DILI, as shown in clinically diagnosed parameters. Interestingly, plasma Hsp90 content was increased time-dependently in APAPtreated patients, in which the findings might indicate potential value of Hsp90 in early DILI screening in addition to other clinical references. In order to validate proposed hypothesis, further studies in vivo and in vitro warrant to be conducted, respectively.

Physiologically, liver cells possess a wide range of biological functions, such as detoxification of metabolites and protein synthesis/secretion. In addition, the liver exert a vital biofunction in metabolism, including hormone production, decomposition of toxin, and glycogen storage [22, 23]. Liver damage can be diagnosed through liver function tests from blood samples, which it can be identified by specific biomarkers [24]. Thus, the detoxifcation ability of liver cells can be showed in condition of drug-induced liver damage, such as acetaminophen. As responded, overdose of APAP results in hepatocellular lesions by cascaded events, such as oxidative stress, inflammatory infiltration, or apoptosis [25, 26]. And yet, APAP-induced early liver injury is still less reported, especially in biomolecule screening. The aldo-keto reductase 7A (AKR7A) functions in the detoxification of environmental toxin and other dicarbonyl-containing compounds, thereby being believed as an endogenous cytoprotective agent [27]. B-cell lymphoma 2 (Bcl-2) plays a key bioeffect in maintaining cellular survival and suppressing the activity of pro-apoptotic regulator [28]. A mitogen-activated protein kinase (MAPK) is involved in regulation of cellular responses to biostimuli, including osmotic stress, heat shock and mitogens. More specifically, p44/42MAPK modulates cell functions of gene expression, cell proliferation/differentiation, and cell survival or apoptosis $[29,30]$. Hsp90 refers to a chaperone protein that aids in other molecules to fold properly, and to stabilize proteins against heat stress, and it also regulates a group of proteins required for cell growth [31], as well as aids in intracellular transport, degradation of protein and facilitating cell signaling [32]. Thus, we extrapolate that Hsp90 biomolecule may respond to early liver injury under drug-induced effects. In our current study, clinical data showed increased content of Hsp90 in plasma of APAP-used patients under early liver lesion, which other detectable parameters (liver injury tests in blood) from clinical diagnosis were unchanged significantly. In APAP-dosed mice at respective 2 and 26 hours, Hsp90 contents in sera and liver were increased, while other liver functional biomolecules and metabolic indicants were changed inapparently. In immunoassays, specific markers of hormone (HGF, insulin, renin) in APAP-dosed tissues were normally expressed, and apoptosis-associated proteins of AKR7 A, Bcl-2, p44/42MAPK in grouped liver cells were activated equally. Interestingly, endogenous Hsp90 expression was up-regulated in APAPdosed liver, as highlighted in immunohistochemistry and immunoblot. In further validation, liver cells study in vitro indicated that APAP-exposed cells resulted in elevation of Hsp90 expression in a time-dependent way. Collectively, the preliminary data from clinical study and researches in vivo and in vitro indicated that Hsp90 may serve as a potential biomolecule for screening early liver lesion. 


\section{Cellular Physiology Cell Physiol Biochem 2017;43:1562-1570

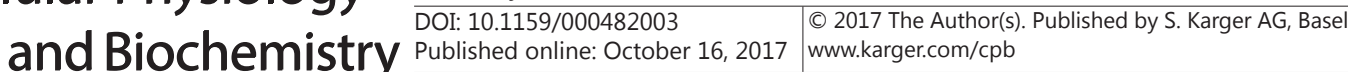

Wu et al.: Bioeffect of Hsp90 on APAP-Treated Liver

\section{Conclusion}

Taken together, the current findings reveal that increased Hsp90 contents is showed in early APAP-induced liver dysfunction, and thus it may be a biomolecule for indicating APAPinduced liver injury. However, more detailed molecular mechanism responsible for Hsp90indicated early DILI needs to be validated in future study.

\section{Acknowledgements}

Our present study is supported by the grants from Talents Highland of Emergency and Medical Rescue of Guangxi Province in China (GXJZ201510, GXJZ201604), National Natural Science Foundation of Guangxi (2016GXNSFBA380055), and National Natural Science Foundation of China (81660091).

\section{Disclosure Statement}

There is no Disclosure Statement that I should disclose.

\section{References}

1 Sarges P, Steinberg JM, Lewis JH: Drug-Induced Liver Injury: Highlights from a Review of the 2015 Literature. Drug Saf 2016;39:801-821.

2 Leise MD, Poterucha JJ, Talwalkar JA: Drug-induced liver injury. Mayo Clin Proc 2014;89:95-106.

7 Licata A: Adverse drug reactions and organ damage: The liver. Eur J Intern Med 2016;28:9-16.

4 Fontana RJ: Acute liver failure including acetaminophen overdose. Med Clin North Am 2008;92:761-794.

5 Lancaster EM, Hiatt JR, Zarrinpar A: Acetaminophen hepatotoxicity: an updated review. Arch Toxicol 2015;89:193-199.

6 Chun LJ, Tong MJ, Busuttil RW, Hiatt JR: Acetaminophen hepatotoxicity and acute liver failure. J Clin Gastroenterol 2009;43:342-349.

7 Vad NM, Yount G, Moore D, Weidanz J, Moridani MY: Biochemical mechanism of acetaminophen (APAP) induced toxicity in melanoma cell lines. J Pharm Sci 2009;98:1409-1425.

8 Schmitt E, Gehrmann M, Brunet M, Multhoff G, Garrido C: Intracellular and extracellular functions of heat shock proteins: repercussions in cancer therapy. J Leukoc Biol 2007;81:15-27.

-9 Chebotareva N, Bobkova I, Shilov E: Heat shock proteins and kidney disease: perspectives of HSP therapy. Cell Stress Chaperones 2017;22:319-343.

10 Liu X, Chen S, Tu J, Cai W, Xu Q: HSP90 inhibits apoptosis and promotes growth by regulating HIF-1 $\alpha$ abundance in hepatocellular carcinoma. Int J Mol Med 2016;37:825-835.

-11 Li R, Guo C, Wu X, Huang Z, Jian C: FGF21 functions as a sensitive biomarker of APAP-treated patients and mice. Oncotarget 2017;8:44440-44446.

12 Guo C, Xie G, Su M, Wu X, Lu X, Wu K, Wei C: Characterization of acetaminophen-induced cytotoxicity in target tissues. Am J Transl Res 2016;8:4440-4445.

13 Li R, Zhang X, Yu L, Zou X, Zhao H: Characterization of Insulin-Immunoreactive Cells and Endocrine Cells Within the Duct System of the Adult Human Pancreas. Pancreas 2016;45:735-742.

14 Li R, Song J, Wu W, Wu X, Su M: Puerarin exerts the protective effect against chemical induced dysmetabolism in rats. Gene 2016;595:168-174.

15 Li R, Liang L, Wu X, Ma X, Su M: Valproate acid (VPA)-induced dysmetabolic function in clinical and animal studies. Clin Chim Acta 2017;468:1-4.

16 Wu K, Guo C, Lu X, Wu X, Pan H, Su M: Impact of perinatal exposure to acetaminophen on hepatocellular metabolic function in offspring. Am J Transl Res 2016;8:5646-5652.

17 Jahr JS, Filocamo P, Singh S: Intravenous acetaminophen: a review of pharmacoeconomic science for perioperative use. Am J Ther 2013;20:189-199. 


\section{Cellular Physiology Cell Physiol Biochem 2017;43:1562-1570 and Biochemistry Published \begin{tabular}{l|l} 
DOI: 10.1159/000482003 & $\begin{array}{l}\text { ( ) 2017 The Author(s). Published by S. Karger AG, Basel } \\
\text { www.karger.com/cpb }\end{array}$
\end{tabular}}

Wu et al.: Bioeffect of Hsp90 on APAP-Treated Liver

18 Hodgman MJ, Garrard AR: A review of acetaminophen poisoning. Crit Care Clin 2012;28:499-516.

19 Bender RP, Lindsey RH Jr, Burden DA, Osheroff N: N-acetyl-p-benzoquinone imine, the toxic metabolite of acetaminophen, is a topoisomerase II poison. Biochemistry 2004;43:3731-3739.

20 Ramachandran A, Jaeschke H: Mechanisms of acetaminophen hepatotoxicity and their translation to the human pathophysiology. J Clin Transl Res 2017;3:157-169.

21 Woolbright BL, Jaeschke H: Role of the inflammasome in acetaminophen-induced liver injury and acute liver failure. J Hepatol 2017;66:836-848.

-22 Miyajima A, Tanaka M, Itoh T: Stem/progenitor cells in liver development, homeostasis, regeneration, and reprogramming. Cell Stem Cell 2014;14:561-574.

-23 Navarro-Alvarez N, Soto-Gutierrez A, Kobayashi N: Hepatic stem cells and liver development. Methods Mol Biol 2010;640:181-236.

24 Wei C, Pan Q Wu K, Li R: Clinical characterization for proliferation and metastasis in advanced hepatocellular carcinoma patients. Int J Clin Exp Pathol 2015;8:13429-13431.

25 Jaeschke H: Acetaminophen: Dose-Dependent Drug Hepatotoxicity and Acute Liver Failure in Patients. Dig Dis 2015;33:464-471.

-26 Yoon E, Babar A, Choudhary M, Kutner M, Pyrsopoulos N: Acetaminophen-Induced Hepatotoxicity: a Comprehensive Update. J Clin Transl Hepatol 2016;4:131-142.

27 Li D, Hinshelwood A, Gardner R, McGarvie G, Ellis EM: Mouse aldo-keto reductase AKR7A5 protects V79 cells against 4-hydroxynonenal-induced apoptosis. Toxicology 2006;226:172-180.

28 Gross A, Katz SG: Non-apoptotic functions of BCL-2 family proteins. Cell Death Differ 2017;24:1348-1358.

29 Zhang Y, Pizzute T, Pei M. A review of crosstalk between MAPK and Wnt signals and its impact on cartilage regeneration. Cell Tissue Res 2014;358:633-649.

-30 Wang H, Li C, Wang Z, Shao Y, Lv Z, Zhang W: p44/42MAPK and p90RSK modulate thermal stressed physiology response in Apostichopus japonicus. Comp Biochem Physiol B Biochem Mol Biol 2016;196:5766.

-31 Tukaj S, Węgrzyn G: Anti-Hsp90 therapy in autoimmune and inflammatory diseases: a review of preclinical studies. Cell Stress Chaperones 2016;21:213-218.

32 Pearl LH: Review: The HSP90 molecular chaperone-an enigmatic ATPase. Biopolymers 2016;105:594-607. 